\title{
Research on Protection and Industrialization Development of Qiang Embroidery Culture in Globalization Context
}

\author{
Zeng Ziyi \\ Art College, Southwest University for Nationalities, Chengdu, Sichuan, China \\ hunter2011@foxmail.com
}

Keywords: Qiang embroidery, Globalization context, Protection, Development

\begin{abstract}
From the Qiang embroidery and Qiang's history, this paper firstly introduces the origin and history background of Qiang embroidery and then elaborates the present situation of qiang embroidery culture in globalization context. Later, this paper analyzes the inheritance and protection problems of Qiang embroidery in earthquake-stricken area. Finally, this paper gives some suggestions of protection and industrialization development of Qiang embroidery culture in globalization context
\end{abstract}

\section{Overview of Protection of Qiang Embroidery Culture in Globalization Context}

History of Qiang and Qiang Embroidery. Aba Tibetan and Qiang Autonomous Prefecture is located in the northwest of Sichuan Province. The earliest record about Qiangwas found in Shang Dynasty more than 3000 years ago. Moreover, the relevant records were very active. Most of the Qiang people in Aba Prefecture in Sichuan Province from at that time migration to inland areas with the upper reaches of the Minjiang River Aboriginal mixed form. There are many kinds of the origin of Qiang embroidery, because it has a long history, it is difficult to verify which one is true. Which one of the most interesting statement is before the period of the Three Kingdoms, the Qiang ethnic minority women and men as heroic, Zhuge Liang sent troops to attack Munsan repeatedly been Qiang women beat. So Ming think of a plan, will be embroidered with peach blossom apron gave Qiang women, this corset with beautiful patterns quickly spread among the women, so Qiang women all day study embroidery art, not intentional training. Legend, of course, can not to be trusted.However, from this legend we can get the knowledge that the culture of Qiang embroidery was originally from the Han nationality of the incoming Qiang. Qiang people added their own understanding and improvement to Han Embroidery style, forming the unique Qiang embroidery culture today.

Present Situation of Qiang Embroidery in Globalization Context. Currently, the protection of Qiang embroidery in Qiang nationality populated areas in China is still relatively complete. But with the acceleration of the process of industrialization, especially in the current rapid development of the globalization of the moment, country folk also showed gradually the trend of assimilation, Qiang gathered this small area is gradually being finished in the whole country atmosphere affected. At the same time, with the rapid development of the current tourism industry, natural scenery and ethnic culture save good Qiang gathering is fast development, become an ideal destination for the country and the people of the world tourism, driven by the economic interests, making the idea of Qiang ethnic minority young people is more utilitarian, very few people to be able to devote themselves to learning Qiang embroidery technology, many special process only older Qiang people will, which makes Qiang embroidery inheritance is facing enormous challenges. At the same time, from the current situation, the Qiang embroidery industry has not formed in the local scale, even if the young people spend a lot of time after the acquisition of Qiang embroidery technology, it is difficult to get a decent income. In the industrialization development of today, the way of the industrial production fast and efficient, and the traditional Qiang embroidery handicraft is more time-consuming, which lead to many of the Qiang young people would rather go out to work or is using machine for processing, and not willing to devote themselves to the study of craftsmanship, which is aggravated the crisis of Qiang embroidery culture cannot be good tradition. Finally, Qiang 
earthquake area has completed the post disaster reconstruction, but local people are still concerned about their own survival and livelihood, trying to improve their living. This kind of environment very easily leads to local people to despise this nationality's traditional culture.After all, the spiritual life is built on the material basis. People's neglecting of the traditional culture is very unfavorable to the development and protection of Qiang embroidery culture.

Inheritance and Protection of Qiang Embroidery. According to the survey, the cultural system of direct economic losses amounted to 1.27 billion Yuan after the Wenchuan earthquake in 2008. There were 53 heritage protection units severely damaged in Aba Tibetan and Qiang Autonomous Prefecture, with the area reaching 83547 square meters. The area includes 18 national key cultural relics protection units, 22 provincial-level cultural relics protection units, 13 state level cultural relics protection units, 2 national heritage, one cultural relic of second national level 1 and 55 cultural relics of third national level damaged, generally 1363 cultural relics damaged, the earthquake caused a total of intangible cultural heritage of people of more than 200 people the injured.Two Shibi heritage artists and five Qiang embroideryartists were killed in the earthquake, the intangible cultural heritage preservation field all 1 completely collapsed, six areas were severely damaged, large quantities of Qiang embroidery art was destroyed in the earthquake. At the same time, the grasp of the present Qiang embroidery special technology more for the elderly, in the earthquake some of the older generation, Qiang embroidery artists were killed in the earthquake, Qiang embroidery technology know how to make a large number of people less, this is a great loss for the inheritance and development of Qiang embroidery, the destruction of the Qiang embroidery culture is almost Disastrous. In the late earthquake, many people care about the inheritance of Qiang embroidery culture, but also for the use of local people to grasp the Qiang embroidery technology, to increase revenue for the local people, so that they can get rid of the dependence on government funding as soon as possible to speed up the reconstruction of their homes, some of the central set up a rescue plan, including the Jet Li rescue fund, one fund and other charitable organizations. These organizations set up the work of the Qiang embroidery rescue plan is currently implemented, the state has set up an art fund, which includes the cultivation of the Qiang embroidery skills, has made it a lot of women to learn the majority of Qiang embroidery technology, and the creation of a number of Qiang embroidery enterprises, at the same time to solve the problem of local people's employment, making Qiang embroidery art has been a rapid succession, and has been part of the embroidery products registered national patent. All enterprise in Wen, Li, Mao three countieshave formed a complete industrial chain. Various charity organizations have been actively help the industry. At the same time, state and local government introduced many policies and helping organizations. For example: "Qiang embroidery helping plan", "women embroidered employment assistance center in Aba Tibetan and Qiang Autonomous Prefecture " and "Qiang cultural ecological protection experimental zone" and so on. Although the post disaster relief work of the Qiang embroidery is progressing well, the future rescuerneeds to be improved.

\section{Problems of Protection of Qiang Embroidery Culture in Globalization Context}

Ideas are Old-fashioned and One-sided. According to the investigation and research, project team found that as the people of Qiang nationalityhave long lived in the Aba Tibetan and Qiang Autonomous Prefecture, people's thinking is still relatively backward. Especially after the earthquake disaster, residents generally want to rebuild their homes as soon as possible, as soon as possible to solve the life of distress, and for the importance of culture in the earthquake is destroyed are clearly not enough. At the same time, the majority of residents of the Qiang embroidery culture understanding only stay in a habit of life, heart think Qiang embroidery process is just making clothing, handicrafts, such as a way of, don't realize Qiang embroidery process is at present our country should focus on the protection, faced with the loss of process of. At the same time, even though a few local people realize the importance of protecting the local Qiang embroidery craft culture, but it also has no better countermeasure, do not know how to protect, to pass on. This requires China's relevant departments and organizations to provide help for the local, not only 
provide basic guarantee for the basic necessities of life, but also instill the concept of advanced culture protection and teaching methods into them.

Protection Management System Remains to be perfected. According to the investigation, project group found that the Qiang embroidery in Qiang nationality populated areas in China lacks of a perfect Qiang embroidery culture protection system, Qiang aggregation across Sichuan, Shaanxi Province, but two, and no specific Qiang cultural protection mechanism, also does not have the relevant professional bodies, part of the details of the Qiang culture collection, collation, recording, investigation work is still relatively weak. Due to the lack of professional and cultural protection agency, protection work efficiency of cultural aspects is low.

Inheritance System needs to be improved. The essence of national culture is the cultural inheritance. If an excellent culture develops without a complete system of inheritance, it is also likely facing the risk of lost. Judging from the survey, Qiang at present about Qiang embroidery inheritance mechanism is still relatively weak. This is mainly because of the Qiang people's quality of life is still not high, in the lack of people's basic living guarantee under the premise, it is difficult to the protection and inheritance of cultural concerns. At the same time, Qiang embroidery culture is lack of effective propaganda, training system, because the propaganda is not enough, so that people for the Qiang embroidery culture is not very understanding, not enough attention. Fewer and fewer people will concern about the skills and less people will grasp the technique, making this unique art and culture facing serious lost risk.

\section{Suggestions of Protection and Industrialization Development of Qiang Embroidery Culture in Globalization Context}

Initially Rescue Female Qiang Victims who badly need Orders. In order to solve the problem of employment of the Qiang nationality after earthquake and to further expand the influence of Qiang embroidery culture, project group according to the market survey found currently on the market the main market for low-end mechanical chemical products in large quantities. Mass production although cannot fully reflect the technical content and details of the Qiang embroidery culture, but for the people of disaster areas to quickly bring profit, to make it as soon as possible to get rid of the various relief gold rely on as soon as possible to achieve self-reliance. Therefore, the early stage of the project should focus on the large quantities of order processing. Mass production mode requires the parties to relief first Qiang people to provide production equipment. At the same time, in order to reflect Qiang embroidery culture style in batch products should work on product design, color, pattern and other factors, so that these external factors can fully reflect the characteristics and the aesthetic sense of the Qiang embroidery.

Develop Foreign Markets and Tourism Industry. The mass production can increase of the existence and the coverage of the Qiang embroidery products. At the same time, itcan play a disguised form of publicity effect. In the process of mass production, the development of foreign markets should also be emphasized. At present foreign markets for Chinese handmade products feeling alone bell, through analysis of embroidery in the foreign market of successful development, can see Qiang embroidery products in the foreign market prospects. At the same time, the production of the Qiang embroidery products should be supplemented by the medium and low living goods, all kinds of small commodities to meet the needs of the mass consumer. The expansion of the way is to combine Qiang products and daily necessities. For example, Qiang embroidered logo embroidered on the bed sheets, pillowcases and towels, handbags, backpacks, clothing and other daily necessities. Secondly, still should develop Qiang cultural tourism industry, making Qiang embroidery tourist souvenirs, tourists to use for the exotic culture sense of curiosity and expand the influence of Qiang embroidery products. Through the tourists to enhance people for the understanding and promotion of Qiang embroidery culture, use of tourism on culture and consumer psychology positive influence, extended sales, Qiang embroidery brand started. Finally, we should make great efforts to develop the middle and low level product market. Then we can use the influence and the profits ofthe bulk products to develop the high level product market. 
Rescue MarginalizedQiang Culture and Qiang Embroidery Artists at a Later Stage. A lot of Qiang embroidery craft heritage people were killed or injured in the earthquake in 2008. The inheritance of Qiang embroidery technology is facing a severe test.Therefore, the most important thing in the process of development of Qiang embroidery culture is to use a variety of ways to collect and Study on the verge of extinction of the Qiang embroidery technology.This process mainly need to several steps. First of all, to the injured successor of Qiang embroidery technology to treat, to recover soon, at the same time, the Qiang embroidery technical inheritance have been killed the relatives to appease, while encouraging the memories of the deceased relatives need to get older skills, to make a long story short Qiang embroidery craft technicians together, to prevent the lost; secondly, the selection of a new batch of young successor organization, young and old technician of a technical team to teach young people as soon as possible, making the acquisition of endangered technology, the need for all ages in the process of inheritance in teach skills training, it can reduce the possibility of lost Technology; finally, new Qiang embroidery heirparticipate in production to expand the industrial scale of Qiang embroidery.

The industrialization of Qiang embroidery is only a measure of disaster relief. It is not the ultimate goal of the Qiang embroidery culture heritage and development. Industry is just a way of this purpose. As the very old non-material cultural heritage, Qiang embroideryis not only a kind of commodity, but also a kind of the Qiang people's spiritual sustenance. It contains the wisdom of Qiang people for thousands years. If you want the Qiang embroidery culture spread into every Chinese people's minds, you need to strengthen the Qiang embroidery culture, such as the establishment of Qiang embroidery skills training base, Qiang embroidery art museum, Qiang embroidery culture experience, and so on. Of course, these measures must be implemented after careful considerationin the earthquakedisaster area. At present, primary task is to help the Qiang people to walk out from the shadow of the earthquake and to enable them to become self-reliant. Based on these approaches, wegradually develop Qiang embroidery industry, making the flowers of Qiang embroidery culture bloom in the world in globalization context.

\section{References}

[1] Y. He. Beautiful QiangEmbroidery Reach Out across Time and Space. East Cang, 2015, (7):1-2.

[2] W. Zhong, Application of Qiang Embroidery in Design of Tourism Textile Product. Packaging Engineering, 2010, (8):23-26.

[3] M. Li, J. Zheng, Trace the Origin of Qiang Embroidery Pattern . Design, 2014, (7):175-176.

[4] J. Xu, G.P. Zhang, Investigation and Analysis on Designs of Embroidery Patterns of Qiang Ethnic Minority. Journal of Silk, 2012, 49, (7):49-54. 